\title{
A shorter immunoperoxidase technique for the demonstration of carcinoembryonic antigen and other cell products
}

\author{
EADIE HEYDERMAN AND A. MUNRO NEVILLE
}

From the Unit of Human Cancer Biology, London Branch, Ludwig Institute for Cancer Research
in conjunction with The Royal Marsden Hospital, Sutton, Surrey SM2 SPX

SUMMARY A reliable immunoperoxidase schedule for the cellular demonstration of tumour andị normal cell products which can be completed in well under two hours is described. The preparations $\vec{\infty}$ are as clear and readable as those stained by routine histological techniques and should form $\mathrm{a}_{\mathrm{O}}$ valuable adjunct in research and diagnostic studies.

The present study was set up to formulate a generally applicable schedule for the cellular demonstration of tumour index substances using routinely prepared histological material by a method which would also be reproducible and readable and allow simultaneous histological diagnosis. The carcinoembryonic antigen (Gold and Freedman, 1965) was used as the prime subject as high-quality reagents were available.

\section{Materials}

Six-millimicron sections of formalin-fixed paraffinembedded material were used. Experiments on methodology were carried out using a moderately differentiated colonic adenocarcinoma and its lymph node metastases. Definitive carcinoembryonic antigen (CEA) studies were then made on 30 colorectal carcinomas, 12 mammary carcinomas, 6 prostatic carcinomas, and 12 non-Hodgkin's lymphomas obtained at surgery or necropsy.

Goat anti-CEA serum (Darcy et al., 1973) absorbed with $10 \mathrm{mg} / \mathrm{ml}$ solubilised human colonic extract at a dilution of 1:200-1:400 and an indirect horse anti-goat peroxidase conjugate prepared by a modification (Birbeck and Robertson, unpublished) of the Nakane and Kawaoi (1974) method was used.

To compare the method with the peroxidase antiperoxidase (PAP) technique (Sternberger et al., 1970; materials from Dakopatts Ltd) experiments were carried out with rabbit anti-CEA serum. Neither of these antisera cross-reacts with CEA (Turberville $e t$ al., 1973).

Received for publication 24 June 1976

\section{Method}

The optimal method, shown in the Table, was reached after a series of experiments designed (1) to decrease the time-consuming nature of previousiy published methods (Primus et al., 1975), (2) to deteff mine a suitable counterstain for histological diagne sis, (3) to decrease non-specific staining due to endogenous peroxidase and/or (4) heterophil antibodies, the stickiness of processed tissues, and $\propto$ proteinaceous reagents, and (5) to evaluate a control $\overrightarrow{\vec{O}}$ system. The principal problem encountered was 3 related to the inhibition of endogenous peroxidase.

ENDOGENOUS PEROXIDASE STAINING

Neither $0.5 \% \mathrm{H}_{2} \mathrm{O}_{2}$ in methanol (Streefkerk, 1972) nor $0.074 \% \mathrm{HCl}$ in ethanol (Weir et al., 1974), nor a 3 . combination of the two, blocked endogenous peroxidase completely: phenylhydrazine, sodium 3 cyanide, sodium azide, and potassium ferri cyanide ${ }_{\circ}$ were ineffective. Although complete inhibition could be achieved with $10 \% \mathrm{H}_{2} \mathrm{O}_{2}$ in methanol (Burns, 1975), which has the advantage of bleaching acid haematin, endothelial staining remained a problem $N$ and could be abolished by $0.1 \mathrm{~m}$ periodic acid as a N blocking agent. The increase in background which results from the creation of sticky aldehyde groups ${ }_{\sigma}^{\omega}$ may be overcome by the use of sodium borohydride? (Nakane method, personal communication). Other? aldehyde blocking agents, such as lysine, are less effective or, like sodium dithionite, result in a loss of specific staining.

As bubbles of nascent hydrogen are produced by $\frac{O}{\mathbb{D}}$ the borohydride which lifts sections with the utmost $\frac{\odot}{\Phi}$ facility, the time (maximum 2 minutes) and concen $\frac{0}{2}$ 


\section{Table Immunoperoxidase method}

+1 Dewax meticulously in xylene; take to water through alcohols.

*2 $2.28 \%(0.1 \mathrm{M})$ periodic acid in distilled water- $5 \mathrm{~min}$.

*3 $0.02 \%$ fresh sodium borohydride in distilled water-2 min

4 Wash off with phosphate buffered saline (PBS), then treat with non-immune serum 1:10 in $1 \%$ bovine serum albumin (horse, sheep or swine-see text) -5 min. Wash off with PBS.

t5 Goat anti-CEA 1:400 or rabbit anti-CEA 1:50-15 min.

†6 Horse anti-goat peroxidase conjugate or sheep anti-rabbit (diluted as required)-15 min.

7 Diaminobenzidine: $100 \mathrm{ml}$ PBS; $50 \mathrm{mg}$ DAB; $100 \mu 130 \% \mathrm{H}_{2} \mathrm{O}$, fresh $-5 \mathrm{~min}$.

8 Counterstain lightly with Mayer's haemalum, blue in lithium carbonate, wash in tap water

9 Dehydrate, clear, and mount in Xam (Gurr).

+To bleach acid haematin After Step 1 Cover with $7 \cdot 5 \% \quad \mathrm{H}_{2} \mathrm{O}_{2}$ in distilled water $-5 \mathrm{~min}$. Wash off thoroughly.

*Wash in running tap water for 5 min after steps 2 and 3.

tWash in slide bath containing PBS pH 7.4 and a few drops of detergent on a mechanical agitator for $15 \mathrm{~min}$ after steps 5 and 6 (indirect conjugate) and after steps $6 \mathrm{a}$ and $6 \mathrm{~b}$ (PAP).

For PAP method Instead of step 6, insert:

†PAP 6a Unlabelled swine anti-rabbit 1:20 then

†PAP 6b Rabbit peroxidase anti-peroxidase immune complex 1:50.

tration $(0.02 \%)$ are critical. Longer reaction timesand higher concentrations are unnecessary (Lillie and Pizzolato, 1972; Culling, 1974). The use of an adhesive such as egg albumen is essential.

While $\mathrm{H}_{2} \mathrm{O}_{2}$ has the advantage of bleaching acid haematin and so may be required in very haemorrhagic tumours, the periodic acidborohydride reaction gives a crispness and clarity to the final preparation with an apparent enhancement of specific staining not found with other methods.

\section{BACKGROUND STAINING}

This proved to be far less of a problem with the indirect immunoperoxidase method than with the PAP method which was more time-consuming and, in our hands, not significantly more sensitive. It was largely reduced by the use of avid high-quality antisera and pretreatment of sections with 1:10 non-immune serum from the species used to raise the second antibody.

\section{CONTROLS}

The use of diaminobenzidine (DAB) alone, DAB + conjugate or normal sera at dilutions of $1: 50$ and above always resulted in a negative reaction. Hence we believe that the only truly reliable control method necessitates the use in parallel of antisera absorbed with the antigen being studied. Abolition of activity may also be confirmed by radioimmunoassay before and after absorption.

\section{Results}

CEA was demonstrated in all the colorectal carcinomas and their metastases (even when in bone and after decalcification), irrespective of the degree of their histological differentiation. CEA was mainly confined to the brush border but was also intracellular in poorly differentiated carcinomas. Contrary to a previous study (Denk et al., 1972), but in agreement with others (Khoo et al., 1973; Huitric et al., 1976), focal CEA staining of the brush border of normal mucosal cells was found. In 10/12 mammary and $3 / 6$ prostatic carcinomas, CEA was demonstrated either in the cytoplasm or on the membrane of luminal surfaces; none was noted in nonHodgkin's lymphomas.

Using this technique, we have applied the method to the successful cellular demonstration in human tissues of calcitonin (medullary thyroid carcinoma), adrenocorticotrophic hormone (bronchial carcinoid), growth hormone (pituitary), human chorionic gonadotrophin (choriocarcinoma), fibrinogen (pericarditis), Australia antigen (liver), immunoglobulins (plasma cells), muramidase (lymph node histiocytes), and $\alpha_{1}$-antitrypsin (liver). Success was not achieved with antinuclear factor in formalin-fixed tissue.

Thanks are due to $\mathrm{Mr}$ Robertson who made the conjugates; to $\mathrm{Mr} \mathrm{J}$. Wilson who independently validated the methods; to $\mathrm{Mr}$ Birbeck and Mrs Cartwright for much help and valuable criticism; and to the staff of the Histopathology Department who cut the many sections required for the study.

\section{References}

Burns, J. (1975). Background staining and sensitivity of the unlabelled antibody-enzyme (PAP) method. Comparison with the peroxidase labelled antibody sandwich method using formalin fixed paraffin embedded material. Histochemistry, 43, 291-294.

Culling, C. F. A. (1974). Handbook of Histopathological and Histochemical Techniques. Butterworth, London.

Darcy, C. A., Turberville, C., and James, R. (1973). Immunological study of carcinoembryonic antigen (CEA) and a related glycoprotein. Brit. J. Cancer, 28, 147-160.

Denk, H., Tappeiner, G., Eckerstorfer, R., and Holzner, J. H. (1972). Carcinoembryonic antigen (CEA) in gastrointestinal and extragastrointestinal tumors and its relationship to tumor-cell differentiation. Int. J. Cancer, 10, 262-272.

Gold, P. and Freedman, S. O. (1965). Demonstration of tumor-specific antigens in human colonic carcinomata by immunological tolerance and absorption techniques.J. exp. Med., 121, 439-462.

Huitric, E., Laumonier, R., Burtin, P., von Kleist, S., and Chavanel, G. (1976). An optical and ultrastructural study of the localization of carcinoembryonic antigen (CEA) in normal and cancerous human rectocolonic mucosa. Lab. Invest., 34/1, 97-107.

Khoo, S. K., Warner, N. L., Lie, J. T., and Mackay, I. R. (1973). Carcinoembryonic antigenic activity of tissue extracts: a quantitative study of malignant and benign neoplasms, cirrhotic liver, normal adult and fetal organs. Int. J. Cancer, 11, 681-687.

Lillie, R. D. and Pizzolato, P. (1972). Histochemical use of borohydrides as aldehyde blocking reagents. Stain Technol., 47, 13-16. 
Nakane, P. K. and Kawaoi, A. (1974). Peroxidase-labelled antibody. A new method of conjugation. J. Histochem. Cytochem., 22, 1084-1091.

Primus, F. J., Wang, R. H., Sharkey, R. M., and Goldenberg, D. M. (1975). Detection of carcinoembryonic antigen in tissue sections by immunoperoxidase. J. immunol. Meth., 8 , 267-276.

Sternberger, L. A., Hardy, P. H., Jr., Cuculis, J. J., and Meyer, H. G. (1970). The unlabeled antibody enzyme method of immunohistochemistry. Preparation and properties of soluble antigen-antibody complex (horseradish peroxidase-antihorseradish peroxidase) and its use in identification of spirochetes. J. Histochem. Cytochem., 18, 315-333.
Streefkerk, J. G. (1972). Inhibition of erythrocyte pseudoperoxidase activity by treatment with hydrogen peroxide following methanol. J. Histochem. Cytochem., 20, 829-831. Turberville, C., Darcy, D. A., Laurence, D. J. R., Johns, E. W., and Neville, A. M. (1973). Studies on carcinoembryonic antigen (CEA) and a related glycoprotein CCEA-2. Preparation and chemical characterisation. Immunochemistry, 10, 841-843.

Weir, E. E., Pretlow, T. G., Pitts, A., and Williams, E. E. (1974). Destruction of endogenous peroxidase activity in order to locate cellular antigens by peroxidase-labeled antibodies. J. Histochem. Cytochem., 22, 51-54. 